J. Korean Math. Soc. 51 (2014), No. 1, pp. 189-202

http://dx.doi.org/10.4134/JKMS.2014.51.1.189

\title{
THE IDENTITY-SUMMAND GRAPH OF COMMUTATIVE SEMIRINGS
}

\author{
Shahabaddin Ebrahimi Atani, Saboura Dolati Pish Hesari, \\ AND MEHDI KHORAMDEL
}

\begin{abstract}
An element $r$ of a commutative semiring $R$ with identity is said to be identity-summand if there exists $1 \neq a \in R$ such that $r+a=1$. In this paper, we introduce and investigate the identity-summand graph of $R$, denoted by $\Gamma(R)$. It is the (undirected) graph whose vertices are the non-identity identity-summands of $R$ with two distinct vertices joint by an edge when the sum of the vertices is 1 . The basic properties and possible structures of the graph $\Gamma(R)$ are studied.
\end{abstract}

\section{Introduction}

One of the associated graphs to a ring $R$ is the zero-divisor graph; it is a simple graph with vertex set $Z(R) \backslash\{0\}$, and two vertices $x$ and $y$ are adjacent if and only if $x y=0$ which is due to Anderson and Livingston [8]. This graph was first introduced by Beck, in [11], where all the elements of $\mathrm{R}$ are considered as the vertices. Since then, there has been a lot of interest in this subject and various papers were published establishing different properties of these graphs as well as relations between graphs of various extensions [3, 8-9, 18, 20-21]. Recently, the study of graphs of rings are extended to include semirings as in [16].

As a generalization of rings, structure of semirings have proven to be useful tools in various disciplines. They have important applications in mathematics and theoretical computer science $[19,22]$. From now on let $R$ be a commutative semiring with identity. We define another graph on $R, \Gamma(R)$, with vertices as elements of $S^{*}(R)=S(R) \backslash\{1\}$ (where $S(R)=\{r \in R: r+a=1$ for some $1 \neq$ $a \in R\})$, where two distinct vertices $a$ and $b$ are adjacent if and only if $a+b=1$. We will make an intensive study on the graph $\Gamma(R)$. We recommend to the reader the references $[1-2,4-7,10,13-15]$ where "+" is used in order to connect edges when $R$ is a commutative ring, however this graph is of a different kind.

Received May 7, 2013; Revised September 5, 2013.

2010 Mathematics Subject Classification. 16Y70, 05C62.

Key words and phrases. I-semiring, co-ideal, $Q$-strong co-ideal, co-semidomain, identitysummand graph, identity-summand element. 
Here is a brief summary of our paper. The main purpose of Section 2 is to investigate some properties of $I$-semirings and its minimal prime co-ideals which be useful in the later sections. In Section 3, we look at the connectedness, the diameter and the girth of the graph $\Gamma(R)$. We completely characterize the diameter and the girth of this graph. Section 4 is devoted when the graph $\Gamma(R)$ is a complete bipartite graph. Indeed, it is shown that for an $I$-semiring $R$, $\Gamma(R)$ is complete bipartite if and only if there exist two distinct prime co-ideals $P_{1}$ and $P_{2}$ of $R$ such that $P_{1} \cap P_{2}=\{1\}$. In Section 5 , we will investigate chromatic number and clique number of the graph $\Gamma(R)$. For example, it is shown that for an I-semiring $R, \chi(\Gamma(R))$ is finite if and only if the co-ideal $\{1\}$ is a finite intersection of prime co-ideals. Section 6 is devoted to study planar property of $\Gamma(R)$. A number of basic results of planar property of $\Gamma(R)$ are given.

In order to make this paper easier to follow, we recall in this section various notions which will be used in the sequel. For a graph $\Gamma$ by $E(\Gamma)$ and $V(\Gamma)$ we denote the set of all edges and vertices, respectively. We recall that a graph is connected if there exists a path connecting any two distinct vertices. The distance between two distinct vertices $a$ and $b$, denoted by $d(a, b)$, is the length of the shortest path connecting them (if such a path does not exist, then $d(a, b)=\infty$ and $d(a, a)=0)$. The diameter of graph $\Gamma$, denoted by $\operatorname{diam}(\Gamma)$, is equal to $\sup \{d(a, b): a, b \in V(\Gamma)\}$. A graph is complete if it is connected with diameter less than or equal to one. We denote the complete graph on $n$ vertices by $K_{n}$. The girth of a graph $\Gamma$, denoted $\mathrm{g}(\Gamma)$, is the length of a shortest cycle in $\Gamma$, provided $\Gamma$ contains a cycle; otherwise; $g(\Gamma)=\infty$. An edge for which the two ends are the same is called a loop at the common vertex. For $r$ a nonnegative integer, an $r$-partite graph is one whose vertex set can be partitioned into $r$ subsets so that no edge has both ends in any one subset. A complete $r$-partite graph is one in which each vertex is joined to every vertex that is not in the same subset. The complete bipartite (i.e., 2-partite) with part sizes $m$ and $n$ is denoted by $K_{m, n}$. We will sometimes call $K_{1, n}$ a star graph. We define a coloring of a graph $G$ to be an assignment of colors (elements of some set) to vertices of $G$, one color to each vertex, so that adjacent vertices are assigned distinct colors. If $n$ colors are used, then the coloring is referred to as an $n$-coloring. If there exists an $n$-coloring of a graph $G$, then $G$ is called $n$-colorable. The minimum $n$ for which a graph $G$ is $n$-colorable is called the chromatic number of $G$ and is denoted by $\chi(G)$. A clique of a graph is its maximal complete subgraph and the number of vertices in the largest clique of graph $G$, denoted by $w(G)$, is called the clique number of $G$.

A commutative semiring $R$ is defined as an algebraic system $(R,+, \cdot)$ such that $(R,+)$ and $(R, \cdot)$ are commutative semigroups, connected by $a(b+c)=$ $a b+a c$ for all $a, b, c \in R$, and there exist $0,1 \in R$ such that $r+0=r, r 0=0 r=0$ and $r 1=1 r=r$ for each $r \in R$. In this paper all semirings considered will be assumed to be commutative semirings with non-zero identity. 
Definition 1.1. Let $R$ be a semiring.

(1) A non-empty subset $I$ of $R$ is called co-ideal, if it is closed under multiplication and satisfies the condition $r+a \in I$ for all $a \in I$ and $r \in R$. A co-ideal $I$ of $R$ is called strong co-ideal provided that $1 \in I$ (clearly, $0 \in I$ if and only if $I=R)[17,19,22]$.

(2) A co-ideal $I$ of $R$ is called subtractive if $x, x y \in I$, then $y \in I$ for all $x, y \in R$ (so every subtractive co-ideal is a strong co-ideal) [17].

(3) A proper co-ideal $P$ of $R$ is called prime if $x+y \in P$, then $x \in P$ or $y \in P$ for all $x, y \in R[17]$.

(4) If $D$ is an arbitrary nonempty subset of $R$, then the set $F(D)$ consisting of all elements of $R$ of the form $d_{1} d_{2} \cdots d_{n}+r$ (with $d_{i} \in D$ for all $1 \leq i \leq n$ and $r \in R$ ) is a co-ideal of $R$ containing $D[17,19,22]$.

(5) A semiring $R$ is called co-semidomain, if $a+b=1(a, b \in R)$, then either $a=1$ or $b=1[17]$.

A strong co-ideal $I$ of a semiring $R$ is called a partitioning strong co-ideal (= $Q$-strong co-ideal) if there exists a subset $Q$ of $R$ such that $R=\bigcup\{q I: q \in Q\}$, where $q I=\{q t: t \in I\}$ and if $q_{1}, q_{2} \in Q$, then $\left(q_{1} I\right) \cap\left(q_{2} I\right) \neq \emptyset$ if and only if $q_{1}=q_{2}$. Let $I$ be a $Q$-strong co-ideal of a semiring $R$ and let $R / I=\{q I: q \in$ $Q\}$. Then $R / I$ forms a semiring under the binary operations $\oplus$ and $\odot$ defined as follows: $\left(q_{1} I\right) \oplus\left(q_{2} I\right)=q_{3} I$, where $q_{3}$ is the unique element in $Q$ such that $\left(q_{1} I+q_{2} I\right) \subseteq q_{3} I$, and $\left(q_{1} I\right) \odot\left(q_{2} I\right)=q_{3} I$, where $q_{3}$ is the unique element in $Q$ such that $\left(q_{1} q_{2}\right) I \subseteq q_{3} I$ (note that $q_{1} I=q_{2} I$ if and only if $\left.q_{1}=q_{2}\right)$. Let $q_{e}$ be the unique element in $Q$ such that $1 \in q_{e} I$. Then $q_{e} I=I$ and $q_{e} I$ is the identity of $R / I[17]$.

\section{Some properties of I-semirings}

In this section we collect some properties concerning $I$-semirings which are useful in the later sections.

Definition 2.1. A semiring $R$ is said to be identity-semiring (= $I$-semiring) if $1+x=1$ for all $x \in R$.

One can easily show that $R$ is an $I$-semiring if and only if $\{1\}$ is a strong co-ideal of $R$.

Example 2.2. (1) Let $X=\{a, b, c\}$ and $R=(P(X), \cup, \cap)$ a semiring with $1_{R}=X$, where $P(X)=$ the set of all subsets of $X$. It is easy to see that $R$ is an $I$-semiring.

(2) Assume that $\mathbb{Z}^{+}$is the set of all non-negative integers and let $R=\mathbb{Z}^{+} \times$ $\mathbb{Z}^{+}$. Then $(R,+, \cdot)$ with the usual operations of addition and multiplication is a semiring which is not an $I$-semiring.

The following proposition gives a source of examples of I-semirings.

Proposition 2.3. If $J$ is a $Q$-strong co-ideal of a semiring $R$, then $R / J$ is an I-semiring. 
Proof. Let $q_{e}$ be the unique element in $Q$ such that $q_{e} J=J$ is the identity in $R / J$. We need to show that $q_{e} J \oplus q J=q_{e} J$ for each $q \in Q$. Since $J$ is a co-ideal of $R, q_{e} J+q J \subseteq q_{e} J$. Let $q_{e} J \oplus q J=q^{\prime} J$, where $q_{e} J+q J \subseteq q^{\prime} J$ for some $q^{\prime} \in Q$. Hence $q^{\prime} J \cap q_{e} J \neq \emptyset$. This shows that $q_{e} J \oplus q J=q_{e} J$.

Definition 2.4. An element $x$ of a semiring $R$ is called co-nilpotent if $n x=1$ for some positive integer $n$. A semiring $R$ is said to be co-reduced semiring if 1 is the only co-nilpotent element of $R$.

We will frequently need the following proposition.

Proposition 2.5. Let $R$ be a commutative I-semiring. Then the following statements hold:

(1) $R$ is co-reduced;

(2) If $J$ is co-ideal, then $J$ is a strong co-ideal of $R$. Moreover, if $x y \in J$, then $x, y \in J$ for every $x, y \in R$. In particular, $J$ is subtractive;

(3) The set $(1: x)=\{r \in R: r+x=1\}$ is a strong co-ideal of $R$ for every $x \in S(R)$.

Proof. (1) Let $x \in R$. We may assume that $x \neq 1$. By assumption, $1+x=1$. If $n x=x+x+\cdots+x=1$, then $x(1+1+\cdots+1)=x=1$ which is a contradiction.

(2) We may assume that $J \neq\{1\}$. So there exists $1 \neq x \in J$. Since $J$ is co-ideal, $1=1+x \in J$. Thus $J$ is a strong co-ideal of $R$. Now let $x y \in J$ $(x, y \in R)$. Then $x+x y \in J$; so $x=x(1+y) \in J$. Similarly, $y \in J$. The "in particular" statement is clear.

(3) Clearly, $1 \in(1: x)$. If $a, b \in(1: x)$, then $a+x=1$ and $b+x=1$, implying $a b+a x+b x+x^{2}=1$. Since $(a b+x)(1+x)(1+a)(1+b)=1$ and $1+x=1+a=1+b=1, a b+x=1$. Thus $a b \in(1: x)$. Let $r \in R$ and $a \in(1: x)$. Since $R$ is an $I$-semiring and $a+x=1, a+r+x=1+r=1$. Thus $a+r \in(1: x)$, as required.

The following example shows that co-reduced semirings are not necessarily $I$-semiring.

Example 2.6. Let $R=\left(\mathbb{Z}^{+},+, \cdot\right)$. It is clear that $R$ is a co-reduced semiring which is not an $I$-semiring.

The remainder of this section is concerned to study the minimal prime coideals of $I$-semirings.

Remark 2.7. Assume that $P$ is a prime co-ideal of a semiring $R$ and let $\sum$ be the set of those prime co-ideals of $R$ which are contained in $P$. Then $P \in \sum$ and the set $\sum$ of prime co-ideals of $R$ (partially ordered by reverse inclusion and using Zorn's Lemma) has at least one minimal element, and any such minimal element of $\sum$ is a prime co-ideal. Thus $P$ has a minimal prime co-ideal.

Theorem 2.8. Let $R$ be an I-semiring. 
(1) If $\left\{P_{\alpha}\right\}_{\alpha \in \Lambda}$ is the set of all prime co-ideals of $R$, then $\cap_{\alpha \in \Lambda} P_{\alpha}=\{1\}$.

(2) If $P_{1}, \ldots, P_{n}$ are the only distinct minimal prime co-ideals of $R$, then $\cap_{i=1}^{n} P_{i}=\{1\}$ and $\{1\} \neq \cap_{1 \leq i \leq n, i \neq j} P_{i}$ for each $1 \leq j \leq n$.

Proof. (1) By Proposition 2.5, it is clear that $\{1\} \subseteq \cap_{\alpha \in \Lambda} P_{\alpha}$. For the reverse inclusion, let $x \in \cap_{\alpha \in \Lambda} P_{\alpha}$. Assume that $x \neq 1$. Set $\sum=\{I: x \notin$ $I, I$ is a co-ideal of $R\}$. Since $\{1\} \in \sum, \sum \neq \emptyset$. Of course, the relation of inclusion, $\subseteq$, is a partial order on $\sum$, and then the partially ordered set $\left(\sum, \subseteq\right)$ has a maximal element by Zorn's Lemma, say $K$. Since $x \notin K, K \neq R$. We claim that $K$ is prime. Let $a+b \in K$ such that $a \notin K$ and $b \notin K$. Since $K$ is properly contained in $F(K \cup\{a\})$ and $F(K \cup\{b\}), x \in F(K \cup\{a\}) \cap F(K \cup\{b\})$. Hence $x=r_{1}+k_{1} a^{n}=r_{2}+k_{2} b^{m}$ for some $r_{1}, r_{2} \in R, k_{1}, k_{2} \in K$ and $n, m \in \mathbb{N}$. Since $k_{1}(a+b)^{n}=k_{1} a^{n}+b t \in K$ for some $t \in R, x+b t=r_{1}+k_{1} a^{n}+b t \in K$. Thus $(x+b)(1+t)=x+b t+x t+b \in K$. By Proposition 2.5, we must have $x+b \in K$. Thus $(b+x)^{2} \in K$. Hence $b^{2}+x=\left(b^{2}+x\right)(1+b)(1+x)(1+b)=$ $(b+x)^{2}+c \in K$, where $c \in R$. Inductively $b^{i}+x \in K$ for each $i \in \mathbb{N}$. In particular $b^{m}+x \in K$. As $k_{2} \in K, k_{2}+x \in K$. Hence $\left(k_{2}+x\right)\left(b^{m}+x\right) \in K$. Therefore $k_{2} b^{m}+x=\left(k_{2} b^{m}+x\right)\left(k_{2}+1\right)(x+1)\left(b^{m}+1\right)=\left(k_{2}+x\right)\left(b^{m}+x\right)+d \in K$, where $d \in R$. So $x+r_{2}+k_{2} b^{m} \in K$; thus $x=x+x \in K$, a contradiction. Therefore $K$ is prime, and so $x \in K$ that is a contradiction. Hence $x=1$, as needed.

(2) Clearly $\cap_{\alpha \in \Lambda} P_{\alpha}=\{1\} \subseteq \cap_{i=1}^{n} P_{i}$. Let $x \in \cap_{i=1}^{n} P_{i}$. If $x \notin \cap_{\alpha \in \Lambda} P_{\alpha}$, then there exists $\alpha \in \Lambda$ such that $x \notin P_{\alpha}$. Obviously, $P_{\alpha}$ is not minimal (because $x \notin P_{\alpha}$ ). By Remark 2.7, $P_{\alpha}$ contains a minimal prime co-ideal $P^{\prime}$ of $R$. Since $P^{\prime}$ is minimal, $P^{\prime}=P_{i}$ for some $1 \leq i \leq n$. Thus $x \in P^{\prime} \subseteq P_{\alpha}$ a contradiction. Therefore $x \in \cap_{\alpha \in \Lambda} P_{\alpha}$. Hence $\cap_{\alpha \in \Lambda} P_{\alpha}=\{1\}=\cap_{i=1}^{n} P_{i}$. Now let $\{1\}=\cap_{1 \leq i \leq n, i \neq j} P_{i}$ for some $1 \leq j \leq n$, so $\cap_{1 \leq i \leq n, i \neq j} P_{i} \subseteq P_{j}$. Since for each $i \neq j, P_{i} \nsubseteq P_{j}$, there is a $x_{i} \in P_{i}$ such that $x_{i} \notin P_{j}$. As $\sum_{i \neq j} x_{i} \in \cap_{1 \leq i \leq n, i \neq j} P_{i} \subseteq P_{j}$, it is easy to see that $x_{i} \in P_{j}$ for some $i \neq j$, which is a contradiction. Therefore $P_{i} \subseteq P_{j}$ for some $1 \leq i \leq n$. Thus we have a contradiction to the minimality of $P_{j}$, and hence $\{1\} \neq \cap_{1 \leq i \leq n, i \neq j} P_{i}$ for each $1 \leq j \leq n$.

\section{Examples and basic structure of $\Gamma(R)$}

We start this section with the following proposition.

Proposition 3.1. Let $R$ be a semiring. Then $\Gamma(R)=\emptyset$ if and only if $R$ is a co-semidomain.

Proof. This follows directly from the definitions.

Here we consider the following question: If $R$ is a semiring, is $\Gamma(R)$ connected? The following is an example of a semiring $R$ where $\Gamma(R)$ is not connected. 
Example 3.2. Assume that $\mathbb{Z}^{+}$is the set of all nonnegative integers and let $R=\mathbb{Z}^{+} \times \mathbb{Z}^{+} \times \mathbb{Z}^{+}$. Then $(R,+, \cdot)$ with the usual operations is a semiring and $S^{*}(R)=\{(1,0,0),(0,1,0),(0,0,1),(0,1,1),(1,0,1),(1,1,0)\}$. An inspection will show that $\Gamma(R)$ is not connected.

It is important for us to be able to recognize when the identity-summand graph of semirings are connected. The concept of " $R$ is an $I$-semiring" helps us to do this. The next theorem gives a more explicit description of the diameter of this graph.

Theorem 3.3. Let $R$ be an I-semiring. Then the following statements hold:

(1) $\Gamma(R)$ is a connected graph with $\operatorname{diam}(\Gamma(R)) \leq 3$;

(2) $\Gamma(R)$ contains no loop;

(3) If $\left|S^{*}(R)\right| \geq 3$, then $\Gamma(R)$ is not a complete graph;

(4) If $\left|S^{*}(R)\right| \geq 3$, then $\operatorname{diam}(\Gamma(R))=2$ or 3 .

Proof. (1) Let $x, y \in S^{*}(R)$ be distinct. If $x+y=1$, then $d(x, y)=1$. So suppose that $x+y \neq 1$. By Proposition $2.5, x+x \neq 1$ and $y+y \neq 1$. Since $x, y \in S^{*}(R), x+a=1$ and $y+b=1$ for some $a, b \in S^{*}(R) \backslash\{x, y\}$. If $a=b$, then $x-a-y$ is a path of length 2 in $\Gamma(R)$. Thus we may assume that $a \neq b$. If $a+b=1$, then $x-a-b-y$ is a path of length 3 , and hence $d(x, y) \leq 3$. If $a+b \neq 1$, then $a+b \neq x$ (otherwise, $x+x=x+a+b=1$, a contradiction). Similarly, $a+b \neq y$. Therefore, $x-a+b-y$ is a path of length 2 . Hence $\operatorname{diam}(\Gamma(R)) \leq 3$.

(2) Follows from Proposition 2.5.

(3) Let $\Gamma(R)$ be complete, and let $a, b$ and $c$ be distinct elements of $S^{*}(R)$. Since $\Gamma(R)$ is complete, $a+b=1$ and $a+c=1$. Thus $b c \in(1: a)$, since $(1: a)$ is a strong co-ideal of $R$ by Proposition 2.5. If $b c=1$, then $c=c(1+b)=$ $c+b c=1$, a contradiction. So assume $b c \neq 1$. Hence $b c \in S^{*}(R)$. If $b c=c$, then $1=b+c=b c+b=b(c+1)=b$ that is a contradiction. So $b c \neq c$. Since $\Gamma(R)$ is complete, $b c+c=1$; hence $c=1$ which is a contradiction. Therefore $\Gamma(R)$ is not complete.

(4) Follows from (1), (2) and (3).

The following is an example of a semiring $R$ where $\Gamma(R)$ is connected but $R$ is not an $I$-semiring.

Example 3.4. Consider the semiring $R=\left(\mathbb{Z}^{+} \times \mathbb{Z}^{+},+, \cdot\right)$. Then $S^{*}(R)=$ $\{(1,0),(0,1)\}$ gives $\Gamma(R)$ is connected but $R$ is not an $I$-semiring.

A cycle of a graph is a path such that the start and end vertices are the same. For a graph $G$, it is well-known that if $G$ contains a cycle, then $\operatorname{g}(G) \leq$ $2 \operatorname{diam}(G)+1$.

Theorem 3.5. Let $R$ be an I-semiring. If $\Gamma(R)$ contains a cycle, then

$$
\mathrm{g}(\Gamma(R)) \leq 4 .
$$


Proof. Suppose that $\Gamma(R)$ contains a cycle. We may assume that $\mathrm{g}(\Gamma(R)) \leq 7$. Suppose that $\mathrm{g}(\Gamma(R))=n$, where $n \in\{5,6,7\}$ and let $x_{1}-x_{2}-\cdots-x_{n}-x_{1}$ be a cycle of minimum length. Since $x_{1}$ is not adjacent to $x_{3}, x_{1}+x_{3} \neq 1$. Let $x_{1}+x_{3} \neq x_{i}$ for $1 \leq i \leq n$. Then $x_{2}-x_{3}-x_{4}-x_{1}+x_{3}-x_{2}$ is a 4 -cycle, a contradiction. Therefore $x_{1}+x_{3}=x_{i}$ for some $1 \leq i \leq n$. We divide the proof into five cases.

Case 1: If $x_{1}+x_{3}=x_{1}$, then $x_{1}-x_{2}-x_{3}-x_{4}-x_{1}$ is a 4-cycle, a contradiction.

Case 2: If $x_{1}+x_{3}=x_{2}$, then $x_{2}-x_{3}-x_{4}-x_{2}$ is a 3-cycle, a contradiction.

Case 3: If $x_{1}+x_{3}=x_{3}$, then $x_{1}-x_{2}-x_{3}-x_{n}-x_{1}$ is a 4-cycle, a contradiction.

Case 4: If $x_{1}+x_{3}=x_{4}$, then $x_{2}-x_{3}-x_{4}-x_{2}$ is a 3-cycle, a contradiction.

Case 5: If $x_{1}+x_{3}=x_{n}$, then $x_{2}-x_{3}-x_{4}-x_{n}-x_{2}$ is a 4-cycle, a contradiction.

Therefore, there must be a shorter cycle in $\Gamma(R)$, and $g(\Gamma(R)) \leq 4$.

A cycle graph is a graph that consists of a single cycle. Our next result characterizes the identity-summand graphs that are a cycle graph.

Theorem 3.6. The only cycle identity-summand graph of a commutative Isemiring is $K_{2,2}$.

Proof. By Theorem 3.3, there is no 3-cycle graph and there are no cycle graphs with five or more vertices by Theorem 3.5. Thus the only cycle graph is $K_{2,2}$.

A vertex $x$ of a connected graph $G$ is a cut-point of $G$ if there are vertices $a$ and $b$ of $G$ such that $x$ is in every path from $a$ to $b$ (and $x \neq a, x \neq b$ ). Equivalently, for a connected graph $G, x$ is a cut-point of $G$ if $G-\{x\}$ is not connected. The following example shows that there exist $I$-semirings $R_{1}$ and $R_{2}$ such that $\Gamma\left(R_{1}\right)$ has cut-points and $\Gamma\left(R_{2}\right)$ is a star graph.

Example 3.7. (1) Let $R_{1}=\{0,1,2,3,5,6,10,15,30\}$. Then $\left(R_{1}\right.$, gcd, lcm) $($ take $\operatorname{gcd}(0,0)=0$ and $\operatorname{lcm}(0,0)=0)$ is an $I$-semiring and $S^{*}\left(R_{1}\right)=\{2,3,5,6$, $10,15\}$. In $\Gamma\left(R_{1}\right)$, an inspection will show that 2,3 and 5 are cut-points.

(2) Let $R_{2}=\{0,1,2,5,10,25,50\}$. Then $\left(R_{2}, \operatorname{gcd}, \mathrm{lcm}\right)($ take $\operatorname{gcd}(0,0)=0$ and $\operatorname{lcm}(0,0)=0)$ is an $I$-semiring. It can be seen than $\Gamma\left(R_{2}\right)$ is a star graph.

\section{Bipartite graphs}

In this section, we want to determine when the identity-summand graph $\Gamma(R)$ is a complete bipartite graph. Now we start with the following definition.

Definition 4.1. Let $P$ be a prime co-ideal of an $I$-semiring $R$. We say that $P$ is an associated prime co-ideal of $R$ precisely when there exists $x \in R$ with $(1: x)=P$.

The set of associated prime co-ideals of $R$ is denoted by $\operatorname{co-} \operatorname{Ass}(R)$. 
Example 4.2. Let $X=\{a, b, c\}$ and $R=(P(X), \cup, \cap)$ a semiring with $1_{R}=$ $X$, where $P(X)=$ the set of all subsets of $X$. Then $S^{*}(R)=\{\{a\},\{b\},\{c\}$, $\{a, b\},\{a, c\},\{b, c\}\}$ and an inspection will show that

$$
\begin{aligned}
& P_{1}=(1:\{a, b\})=\{\{c\},\{b, c\},\{a, c\}, X\}, \\
& P_{2}=(1:\{a, c\})=\{\{b\},\{a, b\},\{c, b\}, X\}, \\
& P_{3}=(1:\{b, c\})=\{\{a\},\{a, c\},\{a, b\}, X\}
\end{aligned}
$$

are prime strong co-ideals of $R$ and co-Ass $(R)=\left\{P_{1}, P_{2}, P_{3}\right\}$.

We are now in a position to show a finer relationship between vertices which are adjacent to all the other vertex and co-Ass of a semiring.

Theorem 4.3. Let $R$ be an I-semiring, and let $a \in S^{*}(R)$ be a vertex of $\Gamma(R)$ which is adjacent to every other vertex in $\Gamma(R)$. Then $(1: a)$ is a maximal element in $\Delta=\left\{(1: x): x \in S^{*}(R)\right\}$ and $(1: a) \in \operatorname{co-} \operatorname{Ass}(R)$.

Proof. Suppose $(1: a)$ is not maximal in $\Delta$. So there is a vertex $x$ in $\Gamma(R)$ such that $(1: a) \subset(1: x)$. Since $a$ is adjacent to every other vertex in $\Gamma(R)$, $x \in(1: a)$. Since $(1: a) \subset(1: x), x=x+x=1$ which is a contradiction. It remains to show that $(1: a)$ is a prime strong co-ideal of $R$. Let $x+y \in(1: a)$ such that $x, y \notin(1: a)$. Thus $x+y+a=1$ and $y+a \neq 1$. So $x, y+a \in S^{*}(R)$. Since $a$ is adjacent to every other vertex in $\Gamma(R)$, we have $a+x=1$ which is a contradiction.

Theorem 4.4. Let $R$ be an I-semiring.

(1) If $\Gamma(R)$ is a complete $r$-partite graph with $r \geq 3$, then at most one part has more than one vertex.

(2) If $\Gamma(R)$ is a complete r-partite graph, then $\Gamma(R)$ is a complete bipartite graph.

Proof. (1) Let $V_{1}, V_{2}, \ldots, V_{r}$ be parts of $\Gamma(R)$. Without loss of generality, let $V_{1}, V_{2}$ have more than one vertex. Let $a \in V_{1}$ and $b \in V_{2}$. Since $r \geq 3$, there exists a part, say $V_{3}$, such that $V_{3} \neq V_{1}$ and $V_{3} \neq V_{2}$. Let $z \in V_{3}$. Since $\Gamma(R)$ is complete $r$-partite, $(1: z) \subseteq(1: a) \cup(1: b)$. Now we show that $(1: z) \subseteq(1: a)$ or $(1: z) \subseteq(1: b)$. Let $x \in(1: z) \backslash(1: a)$ and $y \in(1: z) \backslash(1: b)$. Since $(1: z)$ is a strong co-ideal of $R, x y \in(1: z) \subseteq(1: a) \cup(1: b)$. If $x y \in(1: a)$, then $x, y \in(1: a)$, by Proposition 2.5 which is contradiction. If $x y \in(1: b)$, then $x, y \in(1: b)$, by Proposition 2.5, another contradiction. Thus we may assume that $(1: z) \subseteq(1: a)$. Since $\left|V_{1}\right| \geq 2$, there exists $c \in V_{1}$ with $a \neq c$. Thus $c \in(1: z) \backslash(1: a)$ that is a contradiction.

(2) Let $V_{1}, V_{2}, \ldots, V_{r}$ be parts of $\Gamma(R)$ and $r \geq 3$. By (1), at most one parts of $\Gamma(R)$ has more than one vertex. Let $\left|V_{1}\right|>1$, and $\left|V_{i}\right|=1$ for $2 \leq i \leq r$. Let $a, b \in V_{1}$ and $c_{i} \in V_{i}$. Since $\Gamma(R)$ is a complete $r$-partite graph, $a+c_{3}=1$ and $c_{3}+c_{2}=1$. Since $a, c_{2} \in\left(1: c_{3}\right)$ and $\left(1: c_{3}\right)$ is a strong co-ideal of $R$, $a c_{2} \in\left(1: c_{3}\right) ;$ so $a c_{2}+c_{3}=1$. If $a c_{2}=1$ then $a=a c_{2}+a=1+a=1$, a contradiction. Thus $a c_{2} \in S^{*}(R)$. Since $a c_{2}+a=a \neq 1, a c_{2}, a$ are not 
adjacent. This implies $a c_{2} \in V_{1}$. Since $\Gamma(R)$ is a complete $r$-partite graph, $a c_{2} \in V_{1}$ and $c_{2} \in V_{2}, a c_{2}+c_{2}=1$, where $a c_{2}+c_{2}=c_{2}(a+1)=c_{2}$, which is a contradiction.

Theorem 4.5. Let $R$ be an I-semiring. Then $\Gamma(R)$ is complete bipartite if and only if there exist two distinct prime co-ideals $P_{1}$ and $P_{2}$ of $R$ such that $P_{1} \cap P_{2}=\{1\}$.

Proof. Let $P_{1}, P_{2}$ be two prime co-ideals of $R$ such that $P_{1} \cap P_{2}=\{1\}$. Since $P_{1}+P_{2} \subseteq P_{1} \cap P_{2}, P_{1}+P_{2}=\{1\}$. It follows that $P_{1}=(1: a)$ and $P_{2}=(1: b)$ for each $1 \neq a \in P_{2}, 1 \neq b \in P_{1}$. Thus $P_{1}, P_{2} \in \operatorname{co-Ass}(R)$; hence $P_{1} \cup P_{2} \subseteq S(R)$. Now we show that $S(R) \subseteq P_{1} \cup P_{2}$. If $1 \neq a \in S(R) \backslash P_{1} \cup P_{2}$, then there exists $1 \neq b \in R$ such that $a+b=1 \in P_{1} \cap P_{2}$. Since $P_{1}, P_{2}$ are prime co-ideals of $R$ and $a \notin P_{1} \cup P_{2}, b \in P_{1} \cap P_{2}=\{1\}$, a contradiction. Therefore $S(R)=P_{1} \cup P_{2}$. Set $V_{1}=P_{1} \backslash\{1\}$ and $V_{2}=P_{2} \backslash\{1\}$. Let $a, b \in V_{1}$. If $a+b=1 \in P_{2}$, then $a \in P_{2}$ or $b \in P_{2}$, which is a contradiction. Thus non of elements of $V_{1}$ are adjacent together. Similarly, non of elements of $V_{2}$ are adjacent. This means that $\Gamma(R)$ is a bipartite graph with parts $V_{1}$ and $V_{2}$.

Conversely, let $\Gamma(R)$ be a partite graph with two parts $V_{1}$ and $V_{2}$. First of all, we show that $V_{1} \cup\{1\}$ is a co-ideal of $R$. Let $a, b \in V_{1} \cup\{1\}$. Then $a+c=1$ and $b+c=1$ for each $c \in V_{2} \cup\{1\}$. Since $(1: c)$ is a co-ideal of $R$ by Proposition 2.5 and $a, b \in(1: c), a b \in(1: c)$. So $a b+c=1$. Now let $r \in R$ and $a \in V_{1} \cup\{1\}$. Let $a+r \neq 1$. Since $\Gamma(R)$ is a complete bipartite graph, $a+c=1$ for each $c \in V_{2} \cup\{1\}$. Thus $a+r+c=1$, this means $a+r \in V_{1} \cup\{1\}$. Now we show that $P_{1}=V_{1} \cup\{1\}$ is a prime co-ideal of $R$. Let $a+b \in P_{1}$ such that $a \notin P_{1}$ and $b \notin P_{1}$. Hence $a+(b+c)=1, a+c \neq 1$ and $b+c \neq 1$ for each $c \in V_{2}$. Since $a \notin P_{1}$, so $a \neq 1$ and $a \in V_{2}$. Thus $b+c \in V_{1}$. Since $\Gamma(R)$ is a complete bipartite graph, $b+c=b+c+c=1$ (because $R$ is an $I$-semiring and $c+c=c)$. Since $c \in V_{2}, b \in V_{1}$, a contradiction. Thus $P_{1}$ is a prime strong co-ideal of $R$. Similarly, $P_{2}$ is a prime strong co-ideal of $R$.

Theorem 4.6. Let $R$ be an I-semiring.

(1) If $P_{1}=\left(1: x_{1}\right)$ and $P_{2}=\left(1: x_{2}\right)$ are two distinct elements of co-Ass $(R)$, then we have $x_{1}+x_{2}=1$.

(2) If $\operatorname{co-Ass}(R)=\left\{P_{1}, P_{2}\right\},\left|P_{i}\right| \geq 3(i=1,2)$ and $P_{1} \cap P_{2}=\{1\}$, then $\mathrm{g}(\Gamma(R))=4$.

(3) If $|\operatorname{co-} \operatorname{Ass}(R)| \geq 3$, then $\mathrm{g}(\Gamma(R))=3$.

Proof. (1) By assumption, we may assume that $P_{2} \not P_{1}$. Then there exists $1 \neq a \in P_{2} \backslash P_{1}$ such that $a+x_{2}=1$; so $x_{2} \in P_{1}$ since $P_{2}$ is a prime co-ideal of $R$. Thus $x_{1}+x_{2}=1$.

(2) By Theorem 4.5, $\Gamma(R)$ is a complete bipartite graph with parts $P_{1} \backslash\{1\}$ and $P_{2} \backslash\{1\}$. Since for each $i,\left|P_{i}\right| \geq 3$, we must have $\mathrm{g}(\Gamma(R))=4$.

(3) If $P_{1}=\left(1: x_{1}\right), P_{2}=\left(1: x_{2}\right)$ and $P_{3}=\left(1: x_{3}\right)$ are three distinct elements of co-Ass $(R)$, then $x_{1}-x_{2}-x_{3}-x_{1}$ is a cycle of length 3 in $\Gamma(R)$ by (1), as required. 
For a graph $G$ and vertex $x \in V(G)$, the degree of $x$, denoted $\operatorname{deg}(x)$, is the number of edges of $G$ incident with $x$. For every nonnegative integer $r$, the graph $G$ is called $r$-regular if the degree of each vertex of $G$ is equal to $r$. Also for a given vertex $x \in V(G)$, the neighborhood set of $x$ is the set $N(x)=\{a \in V(G): a$ is adjacent to $x\}$. We need the following lemma proved in [21, Lemma 1.9].

Lemma 4.7. Let $G$ be a finite, simple graph with the property that two distinct vertices $v$ and $w$ of $G$ are non-adjacent if and only if $N(v)=N(w)$. Then $G$ is a complete r-partite graph for some positive integer $r$.

Theorem 4.8. Let $R$ be an I-semiring, and let $\Gamma(R)$ be a finite regular graph. Then $\Gamma(R)$ is $K_{n, n}$ for some $n \in \mathbb{N}$.

Proof. Let $\Gamma(R)$ be a regular graph of degree $n$. If $\Gamma(R)$ contains only two vertices, then $\Gamma(R)=K_{1,1}$. So let $\Gamma(R)$ contains at least 3 vertices. Let $a, b \in$ $S^{*}(R)$ be non-adjacent in $\Gamma(R)$. Since $N(b) \subseteq N(a+b)$ and $\operatorname{deg}(b)=\operatorname{deg}(a+b)$, we have $N(b)=N(a+b)$. By similar argument as before, $N(a)=N(a+b)$; hence $N(a)=N(b)$. Therefore, any two non adjacent vertices on the graph have the same neighborhood, and clearly, the converse is true. So by Lemma 4.7, $\Gamma(R)$ is complete $r$-partite and hence $\Gamma(R)$ is bipartite by Theorem 4.4(2). Since $\Gamma(R)$ is regular, $\Gamma(R)=K_{n, n}$.

Anderson and Mulay [9, Theorem 2.8] proved that for direct product of integral domains and their subrings, the diameter is at most 2 . We generalize this result to identity-summand graph of semirings.

Theorem 4.9. Assume that $R_{1}$ and $R_{2}$ are $I$-co-semidomains and let $R \subseteq$ $R_{1} \times R_{2}$ be an I-semiring with $\left|S^{*}(R)\right| \geq 3$. Then $\Gamma(R)$ is a complete bipartite graph with $\operatorname{diam}(\Gamma(R))=2$.

Proof. Let $\left(r_{1}, r_{2}\right) \in S^{*}(R)$. Then $\left(r_{1}, r_{2}\right)+\left(s_{1}, s_{2}\right)=(1,1)$ for some nonidentity element $\left(s_{1}, s_{2}\right)$ of $R$. By assumption, either $r_{1}=1$ or $s_{1}=1$ and either $r_{2}=1$ or $s_{2}=1$; hence either $r_{1}=1$ or $r_{2}=1$. Set $V_{1}=\left\{(1, a): 1 \neq a \in R_{2}\right\}$ and $V_{2}=\left\{(b, 1): 1 \neq b \in R_{1}\right\}$. We show that any two elements of $V_{1}$ are non-adjacent. Suppose, on the contrary, that $x=\left(1, a_{1}\right), y=\left(1, a_{2}\right) \in V_{1}$ are adjacent in $\Gamma(R)$. So $x+y=\left(1, a_{1}\right)+\left(1, a_{2}\right)=(1,1)$; hence $a_{1}+a_{2}=1$ which implies $a_{1}=1$ or $a_{2}=1$ (because $R_{2}$ is $I$-co-semidomain), a contradiction. By the similar way, non of elements of $V_{2}$ are adjacent in $\Gamma(R)$. Also for every $x=(1, a) \in V_{1}$ and $y=(b, 1) \in V_{2}, x+y=(1, a)+(b, 1)=(1,1)$ which means every element in $V_{1}$ is adjacent to every element in $V_{2}$. Hence $\Gamma(R)$ is a complete bipartite graph and $\operatorname{diam}(\Gamma(R))=2$.

\section{Chromatic number and clique number of $\Gamma(R)$}

In this section, we will investigate chromatic number and clique number of the graph $\Gamma(R)$. 
Lemma 5.1. Let $R$ be an I-semiring with $w(\Gamma(R))$ finite. Then $R$ has a.c.c on co-ideals of the form $(1: x)$, where $x \in R$.

Proof. Let $\left(1: a_{1}\right) \subseteq\left(1: a_{2}\right) \subseteq \cdots$ be an ascending chain of co-ideals of $R$. Let $x_{i} \in\left(1: a_{i}\right) \backslash\left(1: a_{i-1}\right)$, where $i \geq 2$; so $x_{i}+a_{i-1} \neq 1$. Since $x_{i}+a_{i-1}+x_{j}+a_{j-1}=1$ for each $i \neq j$, the set $\left\{x_{i}+a_{i-1}\right\}_{i \geq 2}$ is a clique. Also, for each $i \neq j, x_{i}+a_{i-1} \neq x_{j}+a_{j-1}$ (if not, by Proposition 2.5, $x_{i}+a_{i-1}=$ $x_{i}+a_{i-1}+x_{i}+a_{i-1}=x_{i}+a_{i-1}+x_{j}+a_{j-1}=1$, a contradiction); thus $w(\Gamma(R))$ is not finite, which is a contradiction.

Theorem 5.2. Let $R$ be an I-semiring. Then the following are equivalent.

(1) $\chi(\Gamma(R))$ is finite;

(2) $w(\Gamma(R))$ is finite;

(3) The co-ideal $\{1\}$ is a finite intersection of prime co-ideals.

Proof. $(1) \Rightarrow(2)$ Clearly $w(G) \leq \chi(G)$ for any graph $G$, so the result is trivial.

$(2) \Rightarrow(3)$ Let $w(\Gamma(R))=n$. By Lemma 5.1 , the partially ordered set $(\Delta, \subseteq)$ has a maximal element, where $\Delta=\{(1: a): a \neq 1, a \in R\}$. Let $\left(1: x_{i}\right)(i \in J)$ be the different maximal members of the set $\Delta$. By a usual argument, for each $i \in J,\left(1: x_{i}\right)$ is prime. Therefore $\left\{x_{i}\right\}_{i \in J}$ is a clique in $\Gamma(R)$ by Theorem 4.6(1). Since $w(\Gamma(R))$ is finite, $J$ is a finite set; we show that $\{1\}=\cap_{i \in J}\left(1: x_{i}\right)$. Let $1 \neq x \in R$. Then $(1: x) \subseteq\left(1: x_{i}\right)$ for some $i \in I$. We claim that $x \notin\left(1: x_{i}\right)$. Otherwise, $x+x_{i}=1$, so $x_{i} \in(1: x) \subseteq\left(1: x_{i}\right)$; hence $1=x_{i}+x_{i}=x_{i}$ which is a contradiction. Therefore $x \notin\left(1: x_{i}\right)$, and so $x \notin \cap_{i \in I}\left(1: x_{i}\right)$. Hence $\cap_{i \in I}\left(1: x_{i}\right) \subseteq\{1\}$, and so we have equality.

$(3) \Rightarrow(1)$ Let $\cap_{i=1}^{n} P_{i}=\{1\}$, where for each $1 \leq i \leq n, P_{i}$ is a prime co-ideal of $R$. If $x$ is adjacent to $y$ in $\Gamma(R)$, then there is no $1 \leq i \leq n$ such that $x \notin P_{i}$ and $y \notin P_{i}$. Therefore we can label any vertex $x$ of $\Gamma(R)$ by $\min \left\{i: x \notin P_{i}\right\}$. This implies that $\chi(\Gamma(R)) \leq n$.

Theorem 5.3. Let $R$ be an I-semiring with $w(\Gamma(R))$ finite. Then $\min (R) \subseteq$ $\operatorname{co}-\operatorname{Ass}(R)$, where $\min (R)$ is the set of minimal prime co-ideals of $R$.

Proof. Let $P \in \min (R)$. By an argument like that in Theorem 5.2, $\{1\}=$ $\cap_{i=1}^{n} Q_{i}$ where $Q_{i} \in \operatorname{co}-\operatorname{Ass}(R)$. Hence $\cap_{i=1}^{n} Q_{i} \subseteq P$. By an argument similar to the proof in Theorem 2.8(2), $Q_{j} \subseteq P$ for some $1 \leq j \leq n$. As $P$ is minimal, $Q_{j}=P$. Thus $P \in \operatorname{co-Ass}(R)$ and so $\min (R) \subseteq \operatorname{co-Ass}(R)$.

Theorem 5.4. Let $R$ be an I-semiring. Then $w(\Gamma(R))=|\min (R)|$.

Proof. First we show that $|\min (R)|$ is finite if and only if $w(\Gamma(R))$ is finite. Let $|\min (R)|$ be finite. Then $\{1\}$ is a finite intersection of prime co-ideals by Theorem 2.8; so by Theorem 5.2,w( $\Gamma(R))$ is finite. Now suppose that $w(\Gamma(R))$ is finite. Hence $\{1\}=\cap_{i=1}^{n} P_{i}$ for some prime co-ideals $P_{i}$ of $R$ by Theorem 5.2. Let $\left\{Q_{\alpha}\right\}_{\alpha \in \Lambda}$ be the set of all minimal prime co-ideals of $R$. For each $\alpha \in \Lambda, 1 \in Q_{\alpha}$, so $\cap_{i=1}^{n} P_{i} \subseteq Q_{\alpha}$ for each $\alpha \in \Lambda$. This implies that $P_{i} \subseteq Q_{\alpha}$ for some $1 \leq i \leq n$. Since $Q_{\alpha}$ is minimal, $P_{i}=Q_{\alpha}$. This gives $\Lambda$ is finite, and so $|\min (R)|$ is finite. 
Let $|\min (R)|=n$. By Theorem 2.8, for each $1 \leq j \leq n$ there exists $x_{j} \in$ $\cap_{1 \leq i \leq n, i \neq j} P_{i}$ with $x_{j} \notin P_{j}$. Since each $P_{i}$ is a co-ideal of $R, x_{i}+x_{j} \in P_{s}$ for each $1 \leq s \leq n$, and so $x_{i}+x_{j}=1$ (because $\{1\}=\cap_{i=1}^{n} P_{i}$ ) for each $1 \leq i \neq j \leq n$; thus $X=\left\{x_{1}, x_{2}, \ldots, x_{n}\right\}$ is a clique in $\Gamma(R)$. Hence $w(\Gamma(R) \geq n$. Now we show that $w(\Gamma(R)) \leq n$. Let $w(\Gamma(R))=m$. Then there is $\left\{y_{1}, \ldots, y_{m}\right\}$ such that it is a clique in $\Gamma(R)$. Since $y_{i} \neq 1$ and $\{1\}=\cap_{1 \leq i \leq n} P_{i}$, there is $1 \leq s \leq n$ such that $y_{i} \notin P_{s}$. If $m>n$, then by Pigeon hole principle, there is at least one $P_{s}(1 \leq s \leq n)$ such that $y_{i} \notin P_{s}$ and $y_{j} \notin P_{s}$ for some $(1 \leq i \neq j \leq m)$. Since $P_{s}$ is prime, $y_{i}+y_{j} \notin P_{s}$ which is a contradiction (because $y_{i}+y_{j}=1 \in P_{s}$ ). Hence $m \leq n$. Thus $w(\Gamma(R))=n$.

Example 5.5. Let $R, P_{1}, P_{2}$ and $P_{3}$ be as in Example 4.2. It is easy to see that $P_{1} \cap P_{2} \cap P_{3}=X=\{1\}, X \neq \cap_{1 \leq i \leq 3, i \neq j} P_{i}$ and $w(\Gamma(R))=3$.

Theorem 5.6. If $R$ is an I-semiring, then $\chi(\Gamma(R))=w(\Gamma(R))$.

Proof. It is clear that $w(\Gamma(R)) \leq \chi(\Gamma(R))$. Let $w(\Gamma(R))=n$. Then $\{1\}=$ $P_{1} \cap \cdots \cap P_{n}$, where for each $i, P_{i}$ is a minimal prime co-ideal. By an argument like that in the proof of Theorem $5.2((3) \Rightarrow(1)), \chi(\Gamma(R)) \leq n$. Therefore $\chi(\Gamma(R))=w(\Gamma(R))$.

\section{Planar property of $\Gamma(R)$}

A graph is said to be planar if it can be drawn in the plane so that its edges intersect only at their ends. A subdivision of a graph is a graph obtained from it by replacing edges with pairwise internally-disjoint paths. In this section, the planar property of graph $\Gamma(R)$ is investigated.

Theorem 6.1. Let $R$ be an I-semiring.

(1) If $|\min (R)| \geq 5$, then $\Gamma(R)$ is not planar.

(2) If $|\min (R)|=4$, then $\Gamma(R)$ is not planar.

Proof. (1) This follows from Theorem 5.4.

(2) By Theorem 5.4, $w(\Gamma(R))=4$. Thus there exist $x_{1}, x_{2}, x_{3}, x_{4} \in S(R)^{*}$ such that $\left\{x_{1}, x_{2}, x_{3}, x_{4}\right\}$ forms a clique in $\Gamma(R)$. Let $x_{i j}=x_{i} x_{j}$, where $1 \leq$ $i \neq j \leq 4$. Assume that $1 \leq k \neq i, j \leq 4$. Since $x_{i}, x_{j} \in\left(1: x_{k}\right), x_{i j} \in\left(1: x_{k}\right)$. If $x_{i j}=1$, then $x_{i}=x_{i j}+x_{i}=1$ which is a contradiction. This implies that $x_{i j} \in S^{*}(R)$. If $x_{i j}=x_{s}$ for some $1 \leq s \leq 4$, then we split the proof into two cases:

Case 1. If $s=i$, then $x_{i j}+x_{j}=1$. This implies that $x_{j}=1$ which is a contradiction. Similarly, for $s=j$.

Case 2. If $s \neq j$ and $s \neq i$, then $x_{i j}+x_{s}=1$; hence $x_{s}+x_{s}=1$. It follows that $x_{s}=1$ by Proposition 2.5, a contradiction.

Therefore $x_{i j} \notin\left\{x_{1}, x_{2}, x_{3}, x_{4}\right\}$. Let $s \neq k$ and $s, k \in\{1,2,3,4\}-\{i, j\}$. Since $x_{i j}+x_{s}=1$ and $x_{i j}+x_{k}=1$, we have $x_{s}, x_{k} \in\left(1: x_{i j}\right)$; thus $x_{s k} \in(1$ : $\left.x_{i j}\right)$. Set $V_{1}=\left\{x_{1}, x_{2}, x_{12}\right\}$ and $V_{2}=\left\{x_{34}, x_{3}, x_{4}\right\}$. Then $V_{1}$ and $V_{2}$ are two parts of a complete bipartite subgraph of $\Gamma(R)$. Therefore $K_{3,3}$ is a subgraph of $\Gamma(R)$, and so $\Gamma(R)$ is not planar. 
Example 6.2. Let $R=\left\{2^{i} 3^{j} 5^{k}: i \in\{0,1,2,3\}, j \in\{0,1,2,3\}, k \in\{0,1\}\right\} \cup$ $\{0\}$. Then $(R, \operatorname{gcd}, 1 \mathrm{~cm})$ is an $I$-semiring. An inspection shows that $\{2,3,5\}$ is a clique in $\Gamma(R)$ and $w(\Gamma(R))=3$. Hence $|\min (R)|=3$ by Theorem 5.4. Set $V_{1}=\left\{2,2^{2}, 2^{3}\right\}$ and $V_{2}=\left\{3,3^{2}, 3^{3}\right\}$. Then $K_{3,3}$ is a subgraph of $\Gamma(R)$ with two parts $V_{1}$ and $V_{2}$. Hence $\Gamma(R)$ is not planar.

Remark 6.3. Let $R$ be an $I$-semiring. Then:

(1) If $|\min (R)|=1$, then by Theorem 2.8(2), $\{1\}$ is the only minimal prime co-ideal of $R$. Hence $R$ is co-semidomain and so $\Gamma(R)=\emptyset$ by Proposition 3.1.

(2) If $|\min (R)|=2$, then by Theorem $4.5, \Gamma(R)$ is $K_{n, m}$ for some integer $\mathrm{n}, \mathrm{m}$, where $\left|P_{1}\right|-1=n$ and $\left|P_{2}\right|-1=m$. If $n, m \geq 3$, then $\Gamma(R)$ is not planar.

(3) If $|\min (R)| \geq 4$, then by Theorem $6.1, \Gamma(R)$ is not planar

(4) If $R$ is the $I$-semiring as in Example 4.1, then $|\min (R)|=3$ and $R$ is planar. However there exist $I$-semirings that have only three minimal prime co-ideals and their identity-summand graphs are not planar as Example 4.2 shows. It is not entirely clear for us which semirings with $|\min (R)|=3$, the $\Gamma(R)$ is planar.

Acknowledgment. The authors thank the referee for useful suggestions on the first draft of the manuscript.

\section{References}

[1] A. Abbasi and S. Habibi, The total graph of a commutative ring with respect to proper ideals, J. Korean Math. Soc. 49 (2012), no. 1, 85-98.

[2] S. Akbari, D. Kiani, F. Mohammadi, and S. Moradi, The total graph and regular graph of a commutative ring, J. Pure Appl. Algebra 213 (2009), no. 12, 2224-2228.

[3] S. Akbari, H. R. Maimani, and S. Yassemi, When a zero-divisor graph is planar or a complete r-partite graph, J. Algebra 270 (2003), no. 1, 169-180.

[4] D. F. Anderson and A. Badawi, The total graph of a commutative ring, J. Algebra 320 (2008), no. 7, 2706-2719.

[5] — The total graph of a commutative ring, J. Algebra 320 (2008), no. 7, 2706-2719.

[6] - The total graph of a commutative ring without the zero element, J. Algebra Appl. 11 (2012), no. 4, 1250074, 18 pp.

[7] - The generalized total graph of a commutative ring, J. Algebra Appl. 12 (2013), no. 5, 1250212, 18 pp.

[8] D. F. Anderson and P. S. Livingston, The zero-divisor graph of a commutative rings, J. Algebra 217 (1999), no. 2, 434-447.

[9] D. F. Anderson and S. B. Mulay, On the diameter and girth of a zero-divisor graph, J. Pure Appl. Algebra 210 (2007), no. 2, 543-550.

[10] Z. Barati, K. Khashyarmanesh, F. Mohammadi, and K. Nafar, On the associated graphs to a commutative ring, J. Algebra Appl. 11 (2012), no. 2, 1250037, 17 pp.

[11] I. Beck, Coloring of commutative rings, J. Algebra, 116 (1988), no. 1, 208-226.

[12] A. Bondy and U. S. R. Murty, Graph Theory with Applications, American Elsevier, New York, 1976.

[13] T. Chelvam and T. Asir, On the total graph and its complement of a commutative ring, Comm. Algebra, 41 (2013), no. 10, 3820-3835.

[14] — The intersection graph of gamma sets in the total graph I, J. Algebra Appl. 12 (2013), no. 4, 1250198, 18pp. 
[15] - The intersection graph of gamma sets in the total graph II, J. Algebra Appl. 12 (2013), no. 4, 1250199, $14 \mathrm{pp}$

[16] S. Ebrahimi Atani, An ideal-based zero-divisor graph of a commutative semiring, Glas. Mat. Ser. III 44(64) (2009), no. 1, 141-153.

[17] S. Ebrahimi Atani, S. Dolati Pish Hesari, and M. Khoramdel, Strong co-ideal theory in quotients of semirings, J. of Advanced Research in Pure Math. 5 (2013), no. 3, 19-32.

[18] S. Ebrahimi Atani and A. Yousefian Darani, Zero-divisor graphs with respect to primal and weakly primal ideals, J. Korean Math. Soc. 46 (2009), no. 2, 313-325.

[19] J. S. Golan, Semirings and Their Applications, Kluwer Academic Publishers Dordrecht, 1999.

[20] H. R. Maimani, M. R. Pournaki, and S. Yassemi, Zero-divisor graph with respect to an ideal, Comm. Algebra 34 (2006), no. 3, 923-929.

[21] S. Spiroff and C. Wickham, A zero divisor graph determined by equivalence classes of zero divisors, Comm. Algebra 39 (2011), no. 7, 2338-2348.

[22] H. Wang, On rational series and rational language, Theoret. Comput. Sci. 205 (1998), no. 1-2, 329-336.

Shahabaddin Ebrahimi Atani

Faculty of Mathematical Sciences

UNIVERSITY OF GUILAN

P.O. Box 1914, RASHT, Iran

E-mail address: ebrahimi@guilan.ac.ir

Saboura Dolati Pish Hesari

Faculty of Mathematical Sciences

UNIVERSITY OF GUILAN

P.O. Box 1914, RASHT, Iran

E-mail address: saboura_dolati@yahoo.com

MeHdi Khoramdel

Faculty of Mathematical Sciences

UNIVERSITY OF GUILAN

P.O. Box 1914, RASHT, IraN

E-mail address: mehdikhoramdel@gmail.com 